\title{
以均三嗪为核心的三类新型多杂环分子的合成与生物活性
}

\author{
刘亚宁孙晓娜高然李传银 \\ 王静 李益政张成路* \\ (辽宁师范大学化学化工学院 大连 116029)
}

\begin{abstract}
摘要 均三嗪衍生物具有优良的生物活性, 在均三嗪结构中将其它杂环对接引入已成为创制新型药物分子的重要方 法. 首次将 1,2,4-三唑、三唑并噻二唑和 1,2,4-三嗪等药效基团拼合在均三嗪结构中, 设计合成了三类 21 个新型目标分 子, 并通过 IR、 ${ }^{1} \mathrm{H}$ NMR 和 HRMS 等对目标分子进行了结构表征. 首先为研究不同取代基团对药效活性的影响, 利用 4 种不同的三嗪单取代化合物分别与含有苯基和正戊基的中间体缩合，合成了 8 个含有双 1,2,4-三唑的目标分子，同时针 对三唑环上的氨基进行修饰得到目标产物; 其次利用 7 种不同的三嗪双取代化合物，首次将 1,2,4-三嗪和三唑并噻二唑 构筑在 9 个目标分子中. 评价了 21 个目标分子对 Cdc25B 抑制活性. 结果发现 13 个目标分子对 Cdc25B 均表现出良好 的抑制活性, $\mathrm{IC}_{50}$ 值在 $(3.99 \pm 0.80) \sim(0.44 \pm 0.07) \mu \mathrm{g} / \mathrm{mL}$ 之间，其中 6 个目标分子的 $\mathrm{IC}_{50}$ 值均低于阳性参照物 $\mathrm{Na}_{3} \mathrm{VO}_{4}$, 有望成为潜在的抗肿瘤药物.
\end{abstract}

关键词＼cjkstart均三嗪; 1,2,4-三唑; 三唑并噻二唑; 1,2,4-三嗪; $\mathrm{Cdc} 25 \mathrm{~B}$

\section{Synthesis and Bioactivities of Multiheteocyclic Molecules Based on s-Triazine}

\author{
Liu, Yaning \\ Sun, Xiaona \\ Gao, Ran \\ Li, Chuanyin \\ Wang, Jing \\ Li, Yizheng \\ Zhang, Chenglu* \\ (College of Chemistry and Chemical Engineering, Liaoning Normal University, Dalian 116029)
}

\begin{abstract}
Triazine derivatives have good significant biological activities. It has been an important method to develop novel drugs by introduction of other heterocyclic rings onto $s$-triazine. Twenty-one novel target molecules were designed and synthesized by combination of 1,2,4-triazole, triazolo[3,4-b]thiadiazole and 1,2,4-triazine unit with $s$-triazine respectively. The structures of the target molecules were characterized by IR, ${ }^{1} \mathrm{H}$ NMR and HRMS. In order to study the effect of different substituents on the efficacy activities, first, 8 target molecules containing double 1,2,4-triazole unit were synthesized by the condensation of four different mono-substituted $s$-triazines with intermediates containing $n$-pentyl and benzyl, respectively. Meanwhile, 4 target molecules were synthesized by the modification of the amino group. 9 target molecules were also afforded by the reaction of seven disubstituted $s$-triazines with the intermediates. The inhibitory activities of the 21 target molecules against $\mathrm{Cdc} 25 \mathrm{~B}$ were evaluated. The results showed that 13 target molecules exhibited good inhibitory activities against Cdc25B. The $\mathrm{IC}_{50}$ values were between $(3.99 \pm 0.80) \sim(0.44 \pm 0.07) \mu \mathrm{g} / \mathrm{mL}$, wherein the $\mathrm{IC}_{50}$ of 5 target molecules were lower than comparison reference $\mathrm{Na}_{3} \mathrm{VO}_{4}$, which were expected to be potential anticancer drugs.

Keywords $s$-triazine; 1,2,4-triazole; triazolo[3,4-b]thiadiazole; 1,2,4-triazine; Cdc25B
\end{abstract}

癌症已成为导致全球疾病死亡的主要原因之一，迫 切需要研制新的和更安全的对肿瘤细胞具有更广谱毒 性的抗癌剂, 杂环化合物因其结构的多样性和广泛的生 物活性而倍受关注.

在各种人类癌症中，细胞分裂周期 25 (cell division cycle 25, 缩写 $\mathrm{Cdc} 25)$ 磷酸酯酶 B (Cdc25B) 是过度表达 的, 因此 $\mathrm{Cdc} 25 \mathrm{~B}$ 成为抗癌治疗的一个重要靶标, 设计 合成新型 $\mathrm{Cdc} 25 \mathrm{~B}$ 抑制剂无疑具有重要意义. 将两个或 更多杂环药效基团共筑在一个分子中，可提供高度的结 构多样性，并已被证明其在治疗剂方面有广泛应用，成

\footnotetext{
* Corresponding author. E-mail: zhangchenglu@1nnu.edu.cn

Received January 12, 2017; revised March 31, 2017; published online April 13, 2017.

Project supported by the Science and Technology Research Program of Liaoning Provincial Department of Education (No. 2009A426).

辽宁省教育厅科学技术研究(No. 2009A426)资助项目.
} 
为创制新型药物分子的最为有效的方法 ${ }^{[1]}$.

均三嗪衍生物是一类有显著药效的杂环化合物, 包 括针对不同靶标的抗肿瘤活性 ${ }^{[2 \sim 4]}$ 、抗菌 ${ }^{[5]}$ 、抗炎 ${ }^{[6]}$ 、抗 疮活性 ${ }^{[7,8]}$ 等, 其关联深厚的医学应用, 吸引了众多合成 工作者的兴趣. 许多临床药物, 如用于治疗血癌的特里 塔明(Tretamine), 用于肺癌、乳腺癌和卵巢癌等的六甲 蜜胺 $(\mathrm{HMM})^{[9]}$, 均含有均三嗪骨架结构, 因此针对均三 嗪的结构修饰已成为获得活性优良抗癌药物分子最有 效的途径 ${ }^{[10 \sim 15]}$. 1,2,4-三唑也是一类极具吸引力的杂环 化合物, 具有抗菌和抗病毒等生物活性 ${ }^{[16 ~ 20]}$. 如临床药 物特康唑、伊曲康唑、氟康唑、头孢唑啉、利巴韦林、 三唑仑. 阿普唑仑和乙替唑仑等均含有 $1,2,4$-三唑核心 结构, 因此含 1,2,4-三唑的衍生物设计合成已成为近年 药物研究与开发的重点领域之一. 三唑并噻二唑衍生物 也因其抗菌和抗肿瘤]等多样的生物活性而引起了广泛 的关注 ${ }^{[21 ~ 27]}$, 尤其是最新发现具有该活性组块的分子

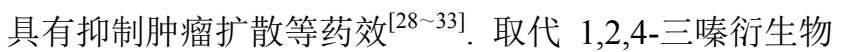
已成为许多药物、合成染料和除草剂中的关键组成, 在 医药和农业领域具有重要的应用前景.

为了探究不同杂环对均三嗪修饰后, 新型分子对 Cdc25B 抑制活性的影响, 基于含 1,2,4-三嗪、1,3,5-三 嗪、1,2,4-三唑和三唑并噻二唑等活性组块分子的重要 应用和本课题组前期研究的成果 ${ }^{[34 ~ 36]}$, 本文以均三嗪 为核心, 分别借助活性硫醚键和亚氨键将上述不同结构 的活性组块对接, 设计了三类迄今未见报道的 21 个新 型多杂环分子, 期望通过评价其对 Cdc25B 的抑制活性, 发现并篎选抗癌药物先导物. 研究中, 首先利用苯胺、 对氯苯胺、吗啉和四氢吡咯修饰均三嗪得单取代中间体
A1 A4，进而分别与 3-苯基-4-氨基-5-巯基-1,2,4-三唑 和 3-正戍基-4-氨基-5-颈基-1,2,4-三唑通过硫醚键对接, 得到目标分子 $1 \sim 8$ (如 Scheme 1 所示); 其次利用烯丙 胺、苯胺、对氯苯胺、吗啉和四氢吡咯修饰均三嗪得双 取代中间体 $\mathbf{A 5} \sim \mathbf{A 9}$ ，进而与 3-氨基-5,6-二苯基-1,2,4三嗪 $(C)$ 通过亚氨基对接，得到目标分子 $9 \sim 13$ (如 Scheme 2 所示). 为了评价三唑环上 4-氨基对活性的影 响, 选择目标分子 7 和 8 , 将分子中的 4-氨基分别利用 苯甲醛和对氯苯甲醛转化成席夫碱, 得到新目标分子 14 17(如 Scheme 3 所示); 最后利用中间体 A10 与 $\mathbf{A 1 1}$ 和中间体 B1 与 B2, 通过缩合反应将均三嗪与三唑并噻 二唑对接, 得到目标分子 $18 \sim 21$ (如 Scheme 4 所示). 在 目标分子设计中，分别利用饱和脂肪含氮杂环、不饱和 脂肪胺和芳香胺对均三嗪修饰，以比较有无 $\pi$ 电子以及 三级胺和二级胺对活性的影响; 在对 1,2,4-三唑环上氨 基修饰时，采用对氯苯基的原因一方面希望借助氯的吸 电性研究电子迁移对活性的影响, 另一方面参考了药物 分子中引入氯原子可增加活性的信息.

\section{1 结果与讨论}

\section{1 目标分子的合成与表征}

IR 谱图中, 在 3100 和 $2900 \mathrm{~cm}^{-1}$ 左右出现苯环上 $\mathrm{C}-\mathrm{H}$ 吸收峰, 1650 和 $1500 \mathrm{~cm}^{-1}$ 左右出现 $\mathrm{C}=\mathrm{N}$ 和 $\mathrm{C}=$ $\mathrm{C}$ 的吸收峰, 在 1150 和 $1100 \mathrm{~cm}^{-1}$ 左右出现 $\mathrm{C}-\mathrm{O}$ 和 $\mathrm{C}-\mathrm{N}$ 吸收峰; ${ }^{1} \mathrm{H}$ NMR 谱图中, $\delta 7.00 \sim 8.00$ 处为芳环的 质子信号, 吗啉环的质子信号在 $\delta 2.8$ 和 3.7 左右, $\mathrm{NH}$ 上 的质子信号在 $\delta 10.0$ 左右处; HRMS 谱图中所有的化合 物均出现了 $[\mathrm{M}+1]^{+}$峰, 结果表明成功合成了目标分子.<smiles>[R]c1nc([R])nc([R2]#N)n1</smiles><smiles>[Y14][R17](Br)(Br)c1nnc(S)n1N</smiles><smiles></smiles>
(A1),<smiles>CNc1ccc(Cl)cc1</smiles><smiles>CN1CCOCC1</smiles><smiles>CN1CCCC1</smiles><smiles>[R]=NCC=CCCCCCCCCCCCNc1ccccc1</smiles><smiles>[Mg][Mg]</smiles><smiles>CNc1ccc(Cl)cc1</smiles><smiles>CN1CCOCC1</smiles>

(A8),<smiles>CN1CCCC1</smiles><smiles>[R][N]Nc1ccccc1</smiles>
$\mathrm{COOH}: \mathrm{R}^{2}=$ Morpholinyl (A10), Pyrryl (A11)

$\mathrm{R}^{4}=\mathrm{Ph}(\mathrm{B} 1), n-\mathrm{C}_{5} \mathrm{H}_{11}$ (B2)

Reagents and condition: (a) (i) $0{ }^{\circ} \mathrm{C}, \mathrm{CH}_{2} \mathrm{Cl}_{2}$, (1) aniline, (2) p-chloroaniline, (3) morphine, (4) nafoxidine, (ii) $10 \sim 20{ }^{\circ} \mathrm{C}, \mathrm{NaOH}-\mathrm{H}_{2} \mathrm{O}, 2.5 \mathrm{~h}$; (b) (i) $4 \sim 5{ }^{\circ} \mathrm{C}, \mathrm{CH}_{2} \mathrm{Cl}_{2}, 0.5 \mathrm{~h}$, (5) ally amine, (6) aniline, (7) p-chloroaniline, (8) morphine, (9) nafoxidine, (ii) $10 \sim 20{ }^{\circ} \mathrm{C}, \mathrm{NaOH}-\mathrm{H}_{2} \mathrm{O}, 2.5 \mathrm{~h}$; (c) p-aminobenzoic acid, $\mathrm{CH}_{3} \mathrm{COOH}, 6 \mathrm{~h}$; (d) THF, $\mathrm{KOH}$, reflux, $8 \mathrm{~h}$

图式 1 目标化合物 $1 \sim 8$ 的合成方法

Scheme 1 Synthetic methods of target compounds $\mathbf{1} \sim \mathbf{8}$ 


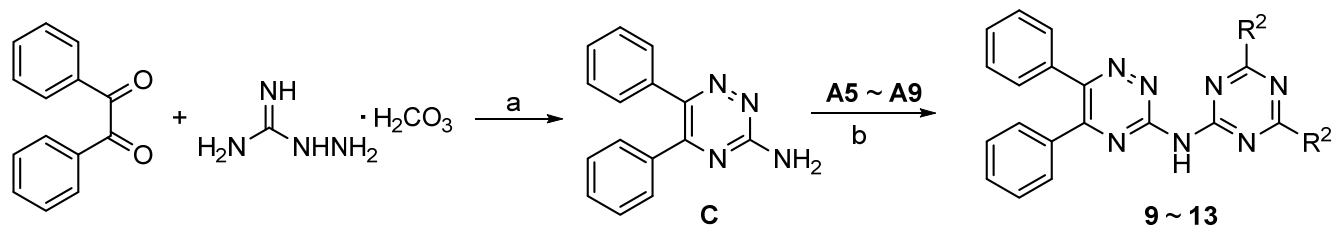

$9 \mathrm{R}^{2}=\mathrm{CH}_{2}=\mathrm{CHCH}_{2} \mathrm{NH}, 10 \mathrm{R}^{2}=\mathrm{PhNH}, 11 \mathrm{R}^{2}=p-\mathrm{ClC}_{6} \mathrm{H}_{4} \mathrm{NH}, 12 \mathrm{R}^{2}=$ Morpholiny, $13 \mathrm{R}^{2}=$ Pyrryl

Reagents and condition: (a) $n-\mathrm{BuOH}$, reflux, $6 \mathrm{~h}$; (b) (i) $0{ }^{\circ} \mathrm{C}$, THF, $\mathrm{NaOH}, 1.5$ h; (ii) r.t, $1 \mathrm{~h}$

图式 2 目标化合物的 9 13 合成方法

Scheme 2 Synthetic methods of target compounds $9 \sim \mathbf{1 3}$

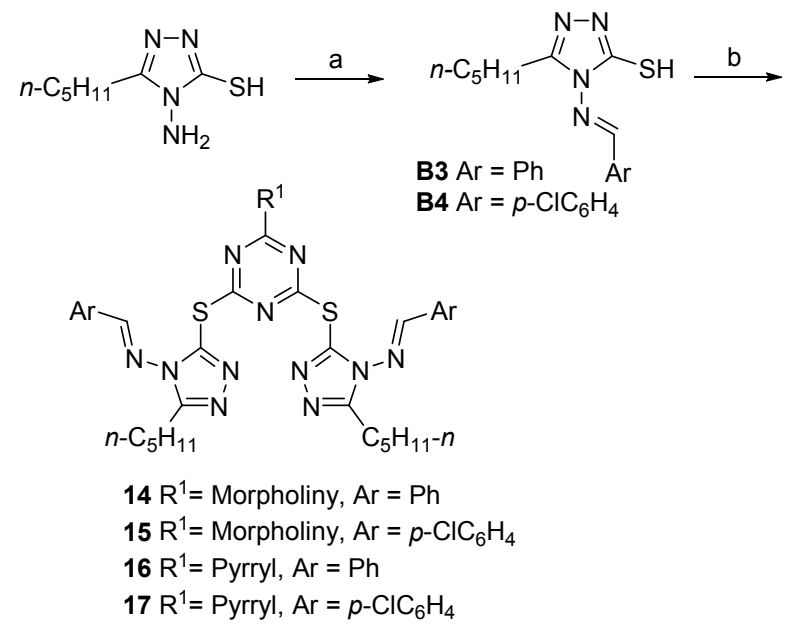

Reagents and condition: (a) benzaldehyde, $p$-chlorobenzaldehyde, $\mathrm{EtOH}$, reflux; (b) THF, KOH, $\mathbf{A} 8$ or $\mathbf{A} 9$, reflux, $24 \mathrm{~h}$

图式 3 目标化合物的 14 17 合成方法

Scheme 3 Synthetic methods of target compounds 14 17<smiles>[R]c1nc([R])nc(Nc2ccc(C(=O)O)cc2)n1</smiles>

图式 4 目标化合物的 18 21 合成方法

Scheme 4 Synthetic methods of target compounds 18 21

\section{2 对 Cdc25B 的抑制活性测试}

目标分子初篮选择的浓度均为 $20 \mu \mathrm{g} / \mathrm{mL}, \mathrm{Na}_{3} \mathrm{VO}_{4}$ 是酪氨酸磷酸酯酶的光谱抑制剂, 可作为 $\mathrm{Cdc} 25 \mathrm{~B}$ 抑制 活性的阳性参照物. 初篮后选择抑制率大于 $50 \%$ 的分子 进行复篮, 得出抑制活性剂量依赖关系, 即 IC50 值. 抑 制活性篮选实验方法参照考文献[37]的方法进行. 本实
验由国家新药中心协助完成, 测试结果见表 1 所示.

表 1 目标分子(TM)对 Cdc25B 的抑制活性 ${ }^{a}$

Table 1 Inhibitory activities of target molecules against Cdc25B

\begin{tabular}{cc||cc||cc}
\hline TM & $\mathrm{IC}_{50} /(\mu \mathrm{g} / \mathrm{mL})$ & TM & $\mathrm{IC}_{50} /(\mu \mathrm{g} / \mathrm{mL})$ & $\mathbf{T M}$ & $\mathrm{IC}_{50} /(\mu \mathrm{g} / \mathrm{mL})$ \\
\hline $\mathbf{1}$ & $\mathrm{NA}^{b}$ & $\mathbf{8}$ & $\mathrm{NA}$ & $\mathbf{1 5}$ & $3.99 \pm 0.80$ \\
$\mathbf{2}$ & $2.63 \pm 0.16$ & $\mathbf{9}$ & $0.97 \pm 0.07$ & $\mathbf{1 6}$ & NA \\
$\mathbf{3}$ & $\mathrm{NA}$ & $\mathbf{1 0}$ & $1.13 \pm 0.42$ & $\mathbf{1 7}$ & $2.46 \pm 0.12$ \\
$\mathbf{4}$ & $\mathrm{NA}$ & $\mathbf{1 1}$ & $2.15 \pm 0.70$ & $\mathbf{1 8}$ & $2.40 \pm 0.11$ \\
$\mathbf{5}$ & $2.77 \pm 0.11$ & $\mathbf{1 2}$ & $\mathrm{NA}$ & $\mathbf{1 9}$ & $0.44 \pm 0.07$ \\
$\mathbf{6}$ & $1.11 \pm 0.05$ & $\mathbf{1 3}$ & $2.23 \pm 0.74$ & $\mathbf{2 0}$ & $0.72 \pm 0.27$ \\
$\mathbf{7}$ & $\mathrm{NA}$ & $\mathbf{1 4}$ & $\mathrm{NA}$ & $\mathbf{2 1}$ & $0.56 \pm 0.06$ \\
Positive control: $\mathrm{Na}_{3} V_{4}$ was for $\mathrm{Cdc} 25 \mathrm{~B}^{{ }^{a} \text { Value tested at } 5 \mu \mathrm{g} / \mathrm{mL}^{\prime}}$ concentration; ${ }^{b}$ not active at $5 \mu \mathrm{g} / \mathrm{mL}$ concentration \\
(inhibition rate $\leqslant 50 \%)$.
\end{tabular}

\section{2 结论}

首次分别将 1,2,4-三唑、三唑并噻二唑和 1,2,4-三嗪 等药效基团拼合在均三嗪结构中，合成了 21 个新型多 杂环目标分子, 其对 Cdc25B 抑制活性结果发现, 13 个 目标分子对 $\operatorname{Cdc} 25 B$ 具有良好的抑制活性, 其中 6 个分 子的 $\mathrm{IC}_{50}$ 值低于阳性参照物 $\mathrm{Na}_{3} \mathrm{VO}_{4}$, 可作为潜在的 Cdc25B 抑制剂, 有望成为抗肿瘤药物先导物.

构效分析发现，12 个双 1,2,4-三唑与均三嗪拼合的 分子中 $(\mathbf{1} \sim \mathbf{8}, \mathbf{1 4} \sim \mathbf{1 7}), 5$ 个对 $\mathrm{Cdc} 25 \mathrm{~B}$ 表现出良好的抑 制活性; 4 个三唑并噻二唑与均三嗪拼合的目标分子 (18 21), 均对 Cdc25B 表现出优秀的抑制活性; 5 个 1,2,4-三嗪与均三嗪拼合的分子 $(\boldsymbol{9} \sim \mathbf{1 3})$ 中, 4 个对 Cdc25B 表现出良好的抑制活性, 表明将双 1,2,4-三唑、 三唑并噻二唑和 1,2,4-三嗪与均三嗪拼合，达到了预期 分子设计的目的, 实现了活性叠加作用. 进一步分析发 现, 以四氢吡咯修饰的均三嗪衍生物活性优于以吗啉修 饰的产物; 对氯苯亚氨基修饰的产物均比苯亚氨基修饰 产物的活性高, 且相比吗啉和四氢吡咯修饰的分子活性 高, 表明当均三嗪环上引入对氯苯亚氨基这一活性药效 基团时，可较好地提高分子的活性. 对比含双 1,2,4-三 唑的目标分子 $(\mathbf{1} \sim \mathbf{8}, \mathbf{1 4} \sim \mathbf{1 7})$, 一方面三唑环 3 -位脂肪 基产物比 3-位芳香基产物活性提高, 可能是因为增大了 
目标分子的脂溶性所致, 另一方氨基修饰产物(14 17) 与 4-氨基未修饰产物 $(\mathbf{5} \sim \mathbf{8})$ 相比, 含有氨基和活性亚氨 基产物 5 和 6, 对比不含活性氨基和活性亚氨基产物 14 和 15, 活性较好, 虽然通过席夫碱结构对电子密度产生 了影响, 但分子中亚氨基 $\mathrm{N}-\mathrm{H}$ 与生物大分子间可能发 生的氢键, 使活性提高, 而且含对氯苯基的分子 $\mathbf{6}$ 和 15 均表现出较好的活性, 验证了预期分子设计中引入氯原 子时可增加活性的判断, 均三嗪利用三级胺修饰时的分 子 7, 8 和 16 活性不佳, 进一步证实了分子中亚氨基 $\mathrm{N}$ $-\mathrm{H}$ 与生物大分子间可能发生的氢键的判断, 由此说明 氢键作用对分子生物活性的重要意义, 氨基未被修饰时 可能因空间障碍对活性促进影响不大. 三类不同结构的 目标分子活性比较发现, 通过亚氨基拼合的分子比通过 硫醚键拼合的分子活性更高, 可能的原因是前者更易与 生物大分子产生氢键.

\section{3 实验部分}

\section{1 仪器与试剂}

AVANCE $500 \mathrm{MHz}$ NMR 核磁共振波谱仪; Waters X evo Q-TOF MS 液相色谱-质谱联用仪; TENSOR 27 傅 立叶变换红外光谱仪; X-5 型数字显微熔点测定仪(温度 计未经校正); WFH-203B 型三用紫外分析仪. 所用的试 剂均为市售的分析纯, 两个测试蛋白 PTP1B、Cdc25B 均由国家新药篮选中心实验室利用大肠杆菌表达并纯 化得到的, 纯度在 $90 \%$ 以上. 化合物 $\mathbf{A 1} \sim \mathbf{A 1 1}, \mathbf{B 3} \sim \mathbf{B} 4$, C 由本课题组自行合成, 合成通法参照文献 [38 40], B1 根据文献[41]合成, 所得结果与文献值相符, B2 参考 文献[42]合成, 收率为 $65.7 \%$, 熔点为 $105.1 \sim 106.4{ }^{\circ} \mathrm{C}$;

\section{2 实验方法}

\subsection{1 目标分子 $1 \sim 8$ 合成通法}

在氮气氛围下, 将 $4 \mathrm{mmol}$ 1,2,4-均三唑(B1, B2)分 别加入到西朗特管中, 加入 $4 \mathrm{~mL}$ 四氢呋喃(THF), 室温 搅拌溶解后加入 $4 \mathrm{mmol} \mathrm{KOH}$ 固体, 滴加 $2 \mathrm{mmol}$ 化合 物(A1 A4) 的 $2 \mathrm{~mL}$ THF 溶液, 室温下搅拌 $0.5 \mathrm{~h}$ 后回流 $6 \mathrm{~h}$. 过滤后得粗产品经乙醇重结晶后得到白色固体产 品 $\mathbf{1} \sim \mathbf{8}$.

6-苯氨基-2,4-(5-硫醚基-4-氨基-3-苯基-1,2,4-三 唑)-1,3,5-三嗪(1): 白色固体, 收率 84\%. m.p. 249.1 $250.2{ }^{\circ} \mathrm{C} ;{ }^{1} \mathrm{H}$ NMR (500 MHz, DMSO- $\left.d_{6}\right) \delta: 10.38(\mathrm{~s}, 1 \mathrm{H}$, $\mathrm{NH}), 8.16 \sim 8.10(\mathrm{~m}, 4 \mathrm{H}, \mathrm{Ph}), 7.55 \sim 7.52(\mathrm{~m}, 6 \mathrm{H}, \mathrm{Ph})$, $7.18(\mathrm{~d}, J=8.00 \mathrm{~Hz}, 2 \mathrm{H}, \mathrm{Ph}), 6.90$ (t, $J=7.65 \mathrm{~Hz}, 2 \mathrm{H}, \mathrm{Ph}$ ), $6.79(\mathrm{t}, J=7.30 \mathrm{~Hz}, 1 \mathrm{H}, \mathrm{Ph}), 6.10\left(\mathrm{~s}, 4 \mathrm{H}, \mathrm{NH}_{2}\right) ;{ }^{13} \mathrm{C} \mathrm{NMR}$ (125 MHz, DMSO- $d_{6}$ ) $\delta: 178.91$ (triazine), 163.87 (triazine), 155.65 (triazole), 145.98 (triazole), $139.35(\mathrm{Ph})$, $130.93(\mathrm{Ph}), 129.93(\mathrm{Ph}), 129.13(\mathrm{Ph}), 128.40(\mathrm{Ph}), 128.05$
(Ph), $123.21(\mathrm{Ph}), 117.89(\mathrm{Ph})$; IR (KBr) v: 3323, 3050, 1618, 1580, 1513, $1292 \mathrm{~cm}^{-1}$; HRMS (positive-ESIMS) calcd for $\mathrm{C}_{25} \mathrm{H}_{21} \mathrm{~N}_{12} \mathrm{~S}_{2}(\mathrm{M}+1)^{+}$553.1382, found 553.1375 .

6-对氯苯氨基-2,4-(5-硫醚基-4-氨基-3-苯基-1,2,4三唑)-1,3,5-三嗪(2): 白色固体, 收率 71\%. m.p. 266.5 $267.8{ }^{\circ} \mathrm{C} ;{ }^{1} \mathrm{H}$ NMR $\left(500 \mathrm{MHz}\right.$, DMSO- $\left.d_{6}\right) \delta: 10.83$ (s, $1 \mathrm{H}$, $\mathrm{NH}), 8.10 \sim 8.02(\mathrm{~m}, 4 \mathrm{H}, \mathrm{Ph}), 7.52 \sim 7.48(\mathrm{~m}, 6 \mathrm{H}, \mathrm{Ph})$, $7.12(\mathrm{~d}, J=8.85 \mathrm{~Hz}, 2 \mathrm{H}, \mathrm{Ph}), 6.89(\mathrm{~d}, J=8.90 \mathrm{~Hz}, 2 \mathrm{H}$, $\mathrm{Ph}), 6.13$ (s, 2H, NH $\left.\mathrm{NH}_{2}\right), 6.07$ (s, $\left.2 \mathrm{H}, \mathrm{NH}_{2}\right) ;{ }^{13} \mathrm{C}$ NMR (125 $\left.\mathrm{MHz}, \mathrm{DMSO}-d_{6}\right) \delta$ : 178.91 (triazine), 163.87 (triazine), 155.65 (triazole), 145.98 (triazole), 137.46 (p-chloroaniline), $130.95(\mathrm{Ph}), 129.93(\mathrm{Ph}), 129.25$ (p-chloroaniline), $128.41(\mathrm{Ph}), 128.36$ ( $p$-chloroaniline), $128.08(\mathrm{Ph}), 121.81$ (p-chloroaniline); IR (KBr) v: 3330, 3037, 1561, 1620, $1582,1515,1292 \mathrm{~cm}^{-1}$; HRMS (positive-ESIMS) calcd for $\mathrm{C}_{25} \mathrm{H}_{20} \mathrm{ClN}_{12} \mathrm{~S}_{2}(\mathrm{M}+1)^{+}$587.0993, found 587.0986.

6- 吗啉-2,4-(5- 颈基 -4- 氨基 -3- 苯基-1,2,4-三 唑)-1,3,5-三嗪(3): 白色固体, 收率 81\%. m.p. 253.7 $254.5{ }^{\circ} \mathrm{C} ;{ }^{1} \mathrm{H}$ NMR (500 MHz, DMSO- $d_{6}$ ) $\delta: 8.04 \sim 8.01$ (m, 4H, Ph), 7.56 7.51 (m, 6H, Ph), $6.05\left(\mathrm{~s}, 4 \mathrm{H}, \mathrm{NH}_{2}\right)$, 3.45 (t, $J=4.70 \mathrm{~Hz}, 4 \mathrm{H}$, morpholine), 3.36 (t, $J=4.70 \mathrm{~Hz}$, $4 \mathrm{H}$, morpholine); ${ }^{13} \mathrm{C}$ NMR (125 MHz, DMSO- $\left.d_{6}\right) \delta$ : 176.68 (triazine), 160.78 (triazine), 154.80 (triazole), 145.53 (triazole), $130.95(\mathrm{Ph}), 129.93(\mathrm{Ph}), 128.40(\mathrm{Ph})$, $128.01(\mathrm{Ph}), 65.16$ (morpholine), 43.25 (morpholine); IR (KBr) $v$ : 3298, 3258, 3103, 2866, 1615, 1583, 1511, 1292, $1252 \mathrm{~cm}^{-1}$; HRMS (positive-ESIMS) calcd for $\mathrm{C}_{23} \mathrm{H}_{23} \mathrm{~N}_{12^{-}}$ $\mathrm{OS}_{2}(\mathrm{M}+1)^{+}$547.1491, found 547.1481.

6-吡咯-2,4-(5- 巯基-4- 氨基-3- 苯基-1,2,4-三 唑)-1,3,5-三嗪(4): 白色固体, 收率 82\%. m.p. 251.8 $252.4{ }^{\circ} \mathrm{C} ;{ }^{1} \mathrm{H}$ NMR $\left(500 \mathrm{MHz}\right.$, DMSO- $\left.d_{6}\right) \delta: 8.06 \sim 8.04$ (m, 4H, Ph), $7.54 \sim 7.50(\mathrm{~m}, 6 \mathrm{H}, \mathrm{Ph}), 6.04\left(\mathrm{~s}, 4 \mathrm{H}, \mathrm{NH}_{2}\right)$, $3.12(\mathrm{t}, J=6.50 \mathrm{~Hz}, 4 \mathrm{H}$, pyrrolidine), $1.86 \sim 1.81(\mathrm{~m}, 4 \mathrm{H}$, pyrrolidine); ${ }^{13} \mathrm{C}$ NMR (125 MHz, DMSO- $d_{6}$ ) $\delta: 176.69$ (triazine), 160.78 (triazine), 154.81 (triazole), 145.53 (triazole), $130.95(\mathrm{Ph}), 129.93(\mathrm{Ph}), 128.40(\mathrm{Ph}), 128.01(\mathrm{Ph})$, 53.61 (pyrrolidine), 24.16 (pyrrolidine); IR (KBr) v: 3230, 3258, 3033, 2867, 1612, 1583, 1512, $1292 \mathrm{~cm}^{-1}$; HRMS (positive-ESIMS) calcd for $\mathrm{C}_{23} \mathrm{H}_{23} \mathrm{~N}_{12} \mathrm{~S}_{2}(\mathrm{M}+1)^{+}$ 531.1541, found 531.1532.

6-苯氨基-2,4-(5-硫醚基-4-氨基-3-正戊基-1,2,4-三 唑)-1,3,5-三嗪(5): 白色固体，收率 78\%. m.p. 239.1 $240.3{ }^{\circ} \mathrm{C} ;{ }^{1} \mathrm{H}$ NMR $\left(500 \mathrm{MHz}\right.$, DMSO- $\left.d_{6}\right) \delta: 10.36(\mathrm{~s}, 1 \mathrm{H}$, $\mathrm{NH}), 7.15$ (d, $J=8.00 \mathrm{~Hz}, 2 \mathrm{H}, \mathrm{Ph}), 6.79$ (t, $J=7.30 \mathrm{~Hz}$, 
$1 \mathrm{H}, \mathrm{Ph}), 6.12\left(\mathrm{~s}, 2 \mathrm{H}, \mathrm{NH}_{2}\right), 2.88\left(\mathrm{t}, J=7.20 \mathrm{~Hz}, 2 \mathrm{H}, \mathrm{CH}_{2}\right)$, 1.80 (t, $\left.J=7.20 \mathrm{~Hz}, 2 \mathrm{H}, \mathrm{CH}_{2}\right), 1.36$ (t, $J=7.00 \mathrm{~Hz}, 4 \mathrm{H}$, $\left.\mathrm{CH}_{2}\right), 0.90\left(\mathrm{t}, J=7.00 \mathrm{~Hz}, 3 \mathrm{H}, \mathrm{CH}_{3}\right) ;{ }^{13} \mathrm{C} \mathrm{NMR}(125 \mathrm{MHz}$, DMSO- $\left.d_{6}\right) \delta$ : 178.90 (triazine), 163.88 (triazine), 155.66 (triazole), 145.97 (triazole), $128.40(\mathrm{Ph}), 128.00(\mathrm{Ph})$, $123.20(\mathrm{Ph}), 117.88(\mathrm{Ph}), 31.22$ (alkyl), 25.81 (alkyl), 25.00 (alkyl), 22.11 (alkyl), 13.88 (alkyl); IR (KBr) v: 3320, 3050, 1616, 1580, 1518, 2940, 2856, 745, 1296 $\mathrm{cm}^{-1}$; HRMS (positive-ESIMS) calcd for $\mathrm{C}_{23} \mathrm{H}_{33} \mathrm{~N}_{12} \mathrm{~S}_{2}$ $(\mathrm{M}+1)^{+}$541.2302, found 541.2311.

6-对氯苯氨基-2,4-(5-硫醚基-4-氨基-3-正戊基1,2,4-三唑)-1,3,5-三嗪(6): 白色固体, 收率 76\%. m.p. $235.5 \sim 236.3{ }^{\circ} \mathrm{C} ;{ }^{1} \mathrm{H}$ NMR (500 MHZ, DMSO-d 6 ) $\delta$ : 10.83 (s, 1H, NH), 7.12 (d, $J=8.85 \mathrm{~Hz}, 2 \mathrm{H}, \mathrm{Ph}), 6.12$ (s, $\left.2 \mathrm{H}, \mathrm{NH}_{2}\right), 2.84\left(\mathrm{t}, J=7.20 \mathrm{~Hz}, 2 \mathrm{H}, \mathrm{CH}_{2}\right), 1.80$ (t, $J=7.20$ $\mathrm{Hz}, 2 \mathrm{H}, \mathrm{CH}_{2}$ ), 1.38 (t, $\left.J=7.00 \mathrm{~Hz}, 4 \mathrm{H}, \mathrm{CH}_{2}\right), 0.88$ (t, $J=$ $\left.7.00 \mathrm{~Hz}, 3 \mathrm{H}, \mathrm{CH}_{3}\right) ;{ }^{13} \mathrm{C}$ NMR (125 MHz, DMSO- $\left.d_{6}\right) \delta$ : 178.99 (triazine), 163.85 (triazine), 155.68 (triazole), 145.98 (triazole), $137.45(\mathrm{Ph}), 129.26(\mathrm{Ph}), 128.36(\mathrm{Ph})$, 121.80 (Ph), 31.22 (alkyl), 25.71 (alkyl), 25.03 (alkyl), 22.15 (alkyl), 13.85 (alkyl); IR (KBr) v: 3330, 3030, 1561, 1620, 1588, 1517, 2949, 2850, 745, $1290 \mathrm{~cm}^{-1}$; HRMS (positive-ESIMS) calcd for $\mathrm{C}_{23} \mathrm{H}_{32} \mathrm{ClN}_{12} \mathrm{~S}_{2}(\mathrm{M}+1)^{+}$ 575.1920 , found 575.1925 .

6-吗啉-2,4-(5-硫醚基-4-氨基-3-正戊基-1,2,4-三 唑)-1,3,5-三嗪(7): 白色固体，收率 79\%. m.p. 241.7 $243.1{ }^{\circ} \mathrm{C} ;{ }^{1} \mathrm{H}$ NMR (500 MHz, DMSO-d ${ }_{6}$ ) $\delta: 6.06$ (s, 2H, $\mathrm{NH}_{2}$ ), 3.48 (t, $J=4.70 \mathrm{~Hz}, 2 \mathrm{H}$, morpholine), 3.36 (t, $J=$ $4.70 \mathrm{~Hz}, 2 \mathrm{H}$, morpholine), 2.89 (t, $J=7.20 \mathrm{~Hz}, 2 \mathrm{H}, \mathrm{CH}_{2}$ ), $1.80\left(\mathrm{t}, J=7.20 \mathrm{~Hz}, 2 \mathrm{H}, \mathrm{CH}_{2}\right), 1.36(\mathrm{t}, J=7.00 \mathrm{~Hz}, 4 \mathrm{H}$, $\mathrm{CH}_{2}$ ), 0.91 (t, $\left.J=7.00 \mathrm{~Hz}, 3 \mathrm{H}, \mathrm{CH}_{3}\right) ;{ }^{13} \mathrm{C} \mathrm{NMR}(125 \mathrm{MHz}$, DMSO- $\left.d_{6}\right) \delta: 176.65$ (triazine), 160.76 (triazine), 154.80 (triazole), 145.51 (triazole), 65.18 (morpholine), 43.26 (morpholine), 31.20 (alkyl), 25.70 (alkyl), 25.05 (alkyl), 22.12 (alkyl), 13.89 (alkyl); IR (KBr) v: 3290, 3255, 3100, 2860, 2944, 2858, 1616, 1583, 1515, 1298, 1254, 745 $\mathrm{cm}^{-1}$; HRMS (positive-ESIMS) calcd for $\mathrm{C}_{21} \mathrm{H}_{35} \mathrm{~N}_{12} \mathrm{OS}_{2}$ $(\mathrm{M}+1)^{+}$535.2430, found 535.2420.

6-吡咯-2,4-(5- 颈基-4- 氨基-3-正戊基 -1,2,4-三 唑)-1,3,5-三嗪(8): 白色固体, 收率 80\%. m.p. 255.5 $256.9{ }^{\circ} \mathrm{C} ;{ }^{1} \mathrm{H}$ NMR (500 MHz, DMSO- $d_{6}$ ) $\delta: 6.19$ (s, 2H, $\mathrm{NH}_{2}$ ), 3.22 (d, $J=4.90 \mathrm{~Hz}, 2 \mathrm{H}$, pyrrolidine), 2.80 (t, $J=$ $7.20 \mathrm{~Hz}, 2 \mathrm{H}, \mathrm{CH}_{2}$ ), 1.88 (t, $J=7.20 \mathrm{~Hz}, 2 \mathrm{H}, \mathrm{CH}_{2}$ ), $1.38 \sim$ $1.35\left(\mathrm{~m}, 2 \mathrm{H}, \mathrm{CH}_{2}\right), 1.33\left(\mathrm{t}, J=7.00 \mathrm{~Hz}, 4 \mathrm{H}, \mathrm{CH}_{2}\right), 0.84(\mathrm{t}$, $\left.J=7.00 \mathrm{~Hz}, 3 \mathrm{H}, \mathrm{CH}_{3}\right) ;{ }^{13} \mathrm{C} \mathrm{NMR}\left(125 \mathrm{MHz}, \mathrm{DMSO}-d_{6}\right) \delta$ :
176.66 (triazine), 160.77 (triazine), 154.83 (triazole), 145.52 (triazole), 53.62 (pyrrolidine), 31.20 (alkyl), 25.71 (alkyl), 25.04 (alkyl), 24.15 (pyrrolidine), 22.11 (alkyl), 13.87 (alkyl); IR (KBr) v: 3233, 3250, 2949, 2850, 1699, 1385, 1279, 876, $746 \mathrm{~cm}^{-1}$; HRMS (positive-ESIMS) calcd for $\mathrm{C}_{21} \mathrm{H}_{35} \mathrm{~N}_{12} \mathrm{~S}_{2}(\mathrm{M}+1)^{+}$519.2455, found 519.2463 .

\section{2 .2 目标化合物 9 13 合成通法}

冰浴下将 $0.155 \mathrm{~g}(0.001 \mathrm{~mol}) 3$-氨基-5,6-二苯基1,2,4-三嗪(C)和 $10 \mathrm{~mL}$ THF 加入三口瓶中, 搅拌溶解后, 加入 $0.08 \mathrm{~g}$ 的 $\mathrm{NaOH}, 0{ }^{\circ} \mathrm{C}$ 下搅拌 $1 \mathrm{~h}$, 将均三嗪衍生物 $\mathbf{A 5} \sim \mathbf{A 9}$ 的 THF 溶液分别逐滴滴加到反应体系中, 摚拌 $30 \mathrm{~min}$, 室温继续反应 $1 \mathrm{~h}$ 后回流反应, 薄层色谱(TLC) 监控反应, 待反应完毕后, 冷却至室温, 过滤, 浓缩滤 液, 用柱层析分离 $(V$ 石油瞇 $: V$ 乙酸醌 $=3: 1)$, 减压蒸除溶 剂, 得到产物 9 13, 收率 53.2\% 73.5\%.

$N, N$-(5,6-二苯基-1,2,4-三嗪-3-基)-(2,4-二烯丙胺基1,3,5-三嗪-6-基)胺(9): 黄色固体，收率 59.7\%. m.p. $176.5 \sim 177.2{ }^{\circ} \mathrm{C} ;{ }^{1} \mathrm{H}$ NMR (500 MHz, DMSO- $\left.d_{6}\right) \delta: 8.92$ (s, 1H, NH), 8.32 (s, 2H, NH), 7.51 (d, J=7.25 Hz, 2H, $\mathrm{PhH}), 7.46$ (d, J=7.45 Hz, 2H, $\mathrm{PhH}), 7.40 \sim 7.37(\mathrm{~m}, 2 \mathrm{H}$, $\mathrm{PhH}), 7.34 \sim 7.25(\mathrm{~m}, 4 \mathrm{H}, \mathrm{PhH}), 5.86 \sim 5.84(\mathrm{~m}, 2 \mathrm{H}$, $\left.\mathrm{CH}_{2} \mathrm{CH}=\mathrm{CH}_{2}\right), 5.13\left(\mathrm{~d}, J=6.25 \mathrm{~Hz}, 4 \mathrm{H}, \mathrm{CH}_{2} \mathrm{CH}=\mathrm{CH}_{2}\right)$, $3.92\left(\mathrm{~d}, J=6.75 \mathrm{~Hz}, 4 \mathrm{H}, \mathrm{CH}_{2} \mathrm{CH}=\mathrm{CH}_{2}\right) ;{ }^{13} \mathrm{C}$ NMR $(125$ $\mathrm{MHz}, \mathrm{DMSO}-d_{6}$ ) $\delta: 165.46$ (triazine, $\mathrm{C} \times 2$ ), 163.93 (triazine), 161.96 (triazine), 157.27 (triazine), 151.13 (triazine), $135.96(\mathrm{Ph}), 135.85\left(\mathrm{CH}_{2}-\mathrm{CH}=\mathrm{CH}_{2}, \mathrm{C} \times 2\right), 135.73(\mathrm{Ph})$, $130.82(\mathrm{Ph}), 129.87(\mathrm{Ph}), 129.37(\mathrm{Ph}, \mathrm{C} \times 2), 129.07(\mathrm{Ph}$, $\mathrm{C} \times 2), 128.60(\mathrm{Ph}, \mathrm{C} \times 2), 128.27(\mathrm{Ph}, \mathrm{C} \times 2), 118.46$ $\left(\mathrm{CH}_{2}-\mathrm{CH}=\mathrm{CH}_{2}, 2 \times \mathrm{C}\right), 48.25\left(\mathrm{CH}_{2}-\mathrm{CH}=\mathrm{CH}_{2}, 2 \times \mathrm{C}\right)$; IR (KBr) v: 3401.4, 3345.5, 3069.3, 2916.8, 1631.4, 1572.8, 1163.3, $731.2 \mathrm{~cm}^{-1}$; HRMS (positive-ESIMS) calcd for $\mathrm{C}_{24} \mathrm{H}_{24} \mathrm{~N}_{9}(\mathrm{M}+1)^{+}$438.2057, found 438.2066.

$N, N$-(5,6-二苯基-1,2,4-三嗪-3-基)-(2,4-二苯胺基1,3,5-三嗪-6-基)胺(10): 浅黄色固体，收率 72.7\%. m.p. $257.3 \sim 258.1{ }^{\circ} \mathrm{C} ;{ }^{1} \mathrm{H}$ NMR (500 MHz, DMSO-d $\left.d_{6}\right) \delta: 8.63$ (s, 3H, NH), 8.09 (t, $J=7.35 \mathrm{~Hz}, 4 \mathrm{H}, \mathrm{PhH}), 7.62 \sim 7.58$ (m, 6H, PhH), 7.47 (d, J=7.25 Hz, 2H, PhH), 7.41 (t, $J=$ $7.75 \mathrm{~Hz}, 2 \mathrm{H}, \mathrm{PhH}), 7.35 \sim 7.29(\mathrm{~m}, 6 \mathrm{H}, \mathrm{PhH}) ;{ }^{13} \mathrm{C}$ NMR $\left(125 \mathrm{MHz}, \mathrm{DMSO}-d_{6}\right) \delta: 168.36$ (triazine, $\mathrm{C} \times 2$ ), 163.63 (triazine), 161.27 (triazine), 157.24 (triazine), 151.63 (triazine), $139.28\left(\mathrm{Ph}^{\prime}, 2 \times \mathrm{C}\right), 136.07(\mathrm{Ph}), 135.83(\mathrm{Ph}), 130.86$ $(\mathrm{Ph}), 130.24(\mathrm{Ph}, \mathrm{C} \times 4), 130.15(\mathrm{Ph}), 129.75(\mathrm{Ph}, \mathrm{C} \times 2)$, $129.31(\mathrm{Ph}, \mathrm{C} \times 2), 128.77(\mathrm{Ph}, \mathrm{C} \times 2), 128.45(\mathrm{Ph}, \mathrm{C} \times 2)$, 122.57 ( $\left.\mathrm{Ph}^{\prime}, \mathrm{C} \times 2\right), 118.76\left(\mathrm{Ph}^{\prime}, \mathrm{C} \times 4\right) ; \mathrm{IR}(\mathrm{KBr}) v$ : 
3047.3, 2938.6, 1634.3, 1590.4, 1072.5, $732.6 \mathrm{~cm}^{-1}$; HRMS (positive-ESIMS) calcd for $\mathrm{C}_{30} \mathrm{H}_{24} \mathrm{~N}_{9}(\mathrm{M}+1)^{+}$ 510.2089, found 510.2086.

$N, N$-(5,6-二苯基-1,2,4-三嗪-3-基)-\{2,4-二(4-氯苯胺 基)-1,3,5-三嗪-6-基\}胺(11): 浅黄色固体，收率 73.2\%. m.p. 285.3 285.9 ${ }^{\circ} \mathrm{C}$; ${ }^{1} \mathrm{H}$ NMR (500 MHz, DMSO- $\left.d_{6}\right) \delta$ : 9.04 (s, 3H, NH), 8.49 (d, $J=6.75 \mathrm{~Hz}, 4 \mathrm{H}, \mathrm{PhH}), 7.65$ (t, $J=6.5 \mathrm{~Hz}, 2 \mathrm{H}, \mathrm{PhH}), 7.59 \sim 7.58(\mathrm{~m}, 2 \mathrm{H}, \mathrm{PhH}), 7.44 \sim$ $7.41(\mathrm{~m}, 2 \mathrm{H}, \mathrm{PhH}), 7.38 \sim 7.33(\mathrm{~m}, 4 \mathrm{H}, \mathrm{PhH}), 6.80$ (dd, $J=1.9 \mathrm{~Hz}, J=6.8 \mathrm{~Hz}, 4 \mathrm{H}, \mathrm{PhH}) ;{ }^{13} \mathrm{C} \mathrm{NMR}(125 \mathrm{MHz}$, DMSO- $\left.d_{6}\right) \delta: 168.35$ (triazine, $\mathrm{C} \times 2$ ), 163.77 (triazine), 161.26 (triazine), 157.17 (triazine), 151.26 (triazine), 138.25 (Ph', C×2), $136.33(\mathrm{Ph}), 136.14(\mathrm{Ph}), 130.96(\mathrm{Ph})$, $130.07(\mathrm{Ph}), 129.83(\mathrm{Ph}, \mathrm{C} \times 2), 129.68(\mathrm{Ph}, \mathrm{C} \times 4)$, $129.54(\mathrm{Ph}, \mathrm{C} \times 2), 128.82(\mathrm{Ph}, \mathrm{C} \times 2), 128.40(\mathrm{Ph}, \mathrm{C} \times 2)$, 127.26 ( $\left.\mathrm{Ph}^{\prime}, \mathrm{C} \times 2\right), 123.65\left(\mathrm{Ph}^{\prime}, \mathrm{C} \times 4\right)$; $\mathrm{IR}(\mathrm{KBr}) v$ : $3089.8,2906.5,1662.4,1582.8,1172.1,728.3 \mathrm{~cm}^{-1}$; HRMS (positive-ESIMS) calcd for $\mathrm{C}_{30} \mathrm{H}_{22} \mathrm{Cl}_{2} \mathrm{~N}_{9}(\mathrm{M}+1)^{+}$ 578.1271, found 578.1277 .

$N, N$-(5,6-二苯基-1,2,4-三嗪-3-基)-(2,4-二吗啉基1,3,5-三嗪-6-基)胺(12): 黄色晶状固体, 收率 70.2\%. m.p. $241.3 \sim 243.2{ }^{\circ} \mathrm{C}$; ${ }^{1} \mathrm{H}$ NMR (500 MHz, DMSO-d $\left.d_{6}\right) \delta$ : $8.74(\mathrm{~s}, 1 \mathrm{H}, \mathrm{NH}), 7.42 \sim 7.36(\mathrm{~m}, 5 \mathrm{H}, \mathrm{PhH}), 7.33 \sim 7.29$ (m, 5H, $\mathrm{PhH}), 3.67$ (t, J=4.15 Hz, 8H, $\left.\mathrm{CH}_{2} \mathrm{NCH}_{2}\right), 3.61(\mathrm{t}$, $\left.J=4.05 \mathrm{~Hz}, 8 \mathrm{H}, \mathrm{CH}_{2} \mathrm{OCH}_{2}\right) ;{ }^{13} \mathrm{C}$ NMR $\left(125 \mathrm{MHz}, \mathrm{CDCl}_{3}\right)$ $\delta: 165.42$ (triazine, $\mathrm{C} \times 2$ ), 163.49 (triazine), 157.74 (triazine, 156.20 (triazine), 152.54 (triazine), $135.91(\mathrm{Ph})$, $135.72(\mathrm{Ph}), 130.68(\mathrm{Ph}), 129.85(\mathrm{Ph}), 129.33(\mathrm{Ph}, \mathrm{C} \times 2)$, $129.05(\mathrm{Ph}, \mathrm{C} \times 2), 128.56(\mathrm{Ph}, \mathrm{C} \times 2), 128.25(\mathrm{Ph}, \mathrm{C} \times 2)$, 66.82 (morpholine), 43.74 (morpholine); IR (KBr) $v$ : 3330.3, 3036.6, 2928.4, 1624.8, 1596.4, $1030.2 \mathrm{~cm}^{-1}$; HRMS (positive-ESIMS) calcd for $\mathrm{C}_{26} \mathrm{H}_{28} \mathrm{~N}_{9} \mathrm{O}_{2}(\mathrm{M}+1)^{+}$ 498.2301, found 498.2298.

$N, N$-(5,6-二苯基-1,2,4-三嗪-3-基)-(2,4-二吡咯烷1,3,5-三嗪-6-基)胺(13): 黄色固体, 收率 73.2\%. m.p. $248.3 \sim 249.6{ }^{\circ} \mathrm{C} ;{ }^{1} \mathrm{H}$ NMR (500 MHz, DMSO- $\left.d_{6}\right) \delta: 8.98$ $(\mathrm{s}, 1 \mathrm{H}, \mathrm{NH}), 7.54 \sim 7.50(\mathrm{~m}, 2 \mathrm{H}, \mathrm{PhH}), 7.38(\mathrm{~d}, J=7.2 \mathrm{~Hz}$, 2H, PhH), $7.30(\mathrm{~d}, J=7.25 \mathrm{~Hz}, 2 \mathrm{H}, \mathrm{PhH}), 6.98 \sim 6.92(\mathrm{~m}$, $4 \mathrm{H}, \mathrm{PhH}), 3.67(\mathrm{t}, J=4.15 \mathrm{~Hz}, 8 \mathrm{H}$, pyrrolidine), $1.85 \sim 1.80$ (m, 8H, pyrrolidine); ${ }^{13} \mathrm{C}$ NMR $\left(125 \mathrm{MHz}, \mathrm{CDCl}_{3}\right) \delta$ : 165.41 (triazine, $\mathrm{C} \times 2$ ), 163.46 (triazine), 157.77 (triazine), 156.22 (triazine), 152.53 (triazine), $135.95(\mathrm{Ph})$, $135.75(\mathrm{Ph}), 130.66(\mathrm{Ph}), 129.88(\mathrm{Ph}), 129.36(\mathrm{Ph}, \mathrm{C} \times 2)$, $129.08(\mathrm{Ph}, \mathrm{C} \times 2), 128.58(\mathrm{Ph}, \mathrm{C} \times 2), 128.26(\mathrm{Ph}, \mathrm{C} \times 2)$, 54.15 (pyrrolidine), 25.22 (pyrrolidine); IR (KBr) v: 3376,
3047.3, 2938, 1634, 1598, 1388, 1270, $733 \mathrm{~cm}^{-1}$; HRMS (positive-ESIMS) calcd for $\mathrm{C}_{26} \mathrm{H}_{28} \mathrm{~N}_{9}(\mathrm{M}+1)^{+}$466.2371, found 466.2379 .

\subsection{3 目标化合物 14 17 合成通法}

将 Schiff 碱(B3 或 B4, $\left.5 \times 10^{-4} \mathrm{~mol}\right)$ 溶于 $10 \mathrm{~mL}$ 的 $\mathrm{THF}$ 中(温度为 $\left.66{ }^{\circ} \mathrm{C}\right)$, 加入 $0.056 \mathrm{~g} \mathrm{KOH}\left(1 \times 10^{-3}\right.$ $\mathrm{mol})$, 搅拌, 溶液由澄清变成黄色浑浊, 加入三嗪单取 代物(A8 或 A9, $2.5 \times 10^{-4} \mathrm{~mol}$ ), 反应 $24 \mathrm{~h}$, 静止, 冷却 至室温, 将固体抽滤, 固体用乙醚洗涤 2 次后用石油 醚: 乙酸乙酯 $(V: V=3: 1)$ 进行柱层析的目标产物.

二- $N$ - 苯亚基-3-((4-)((4-((苯亚基) 氨基 -5- 苯基 4H-1,2,4-三唑-3-基)硫醚)-6-吗啡啉-1,3,5-三嗪-2-基)硫 醚)-5-苯基-4H-1,2,4-三唑-4-胺(14): 浅黄色固体, 收率 70.4\%. m.p. 234.2 235.8 ${ }^{\circ} \mathrm{C} ;{ }^{1} \mathrm{H}$ NMR (500 MHz, $\left.\mathrm{CDCl}_{3}\right) \delta: 8.24(\mathrm{~s}, 1 \mathrm{H}, \mathrm{CH}=\mathrm{N}), 8.00(\mathrm{~d}, J=8.30 \mathrm{~Hz}, 2 \mathrm{H}$, $\mathrm{PhH}), 6.96$ (d, $J=8.30 \mathrm{~Hz}, 3 \mathrm{H}, \mathrm{PhH}), 3.73$ (t, $J=5.00 \mathrm{~Hz}$, $\left.2 \mathrm{H}, \mathrm{CH}_{2} \mathrm{OCH}_{2}\right), 3.55$ (t, J=4.75 Hz, $2 \mathrm{H}, \mathrm{CH}_{2} \mathrm{NCH}_{2}$ ), 2.85 $\left(\mathrm{t}, J=7.20 \mathrm{~Hz}, 2 \mathrm{H}, \mathrm{CH}_{2}\right), 1.81\left(\mathrm{t}, J=7.20 \mathrm{~Hz}, 2 \mathrm{H}, \mathrm{CH}_{2}\right)$, 1.36 (t, $J=7.00 \mathrm{~Hz}, 4 \mathrm{H}, \mathrm{CH}_{2}$ ), 0.90 (t, $J=7.00 \mathrm{~Hz}, 3 \mathrm{H}$, $\left.\mathrm{CH}_{3}\right) ;{ }^{13} \mathrm{C} \mathrm{NMR}\left(125 \mathrm{MHz}, \mathrm{CDCl}_{3}\right) \delta: 176.74$ (triazine), 163.52 (triazole), 162.50 (triazole), 160.70 (triazine), 150.84(N=CH), $130.94(\mathrm{Ph}), 129.92(\mathrm{Ph}), 128.38(\mathrm{Ph})$, 128.00 (Ph), 65.18 (morpholine), 43.23 (morpholine), 31.20 (alkyl), 25.80 (alkyl), 25.01 (alkyl), 22.14 (alkyl), 13.85 (alkyl); IR (KBr) v: 3030, 2940, 2850, 1690, 1600, 1510, 1450, 1375, 1290, 1250, $745 \mathrm{~cm}^{-1}$; HRMS (positive-ESIMS) calcd for $\mathrm{C}_{35} \mathrm{H}_{43} \mathrm{~N}_{12} \mathrm{OS}_{2}(\mathrm{M}+1)^{+} 711.3038$, found 711.3046 .

二- $N$-(4-氯苯亚基)-3-((4-((4-((4-氯苯亚基)氨基-5苯基-4H-1,2,4-三唑-3-基)硫醚)-6-吗啡啉-1,3,5-三嗪-2基)硫醚)-5-苯基-4H-1,2,4-三唑-4-胺(15)：黄色固体，收 率 71.2\%. m.p. 187.8 $188.9{ }^{\circ} \mathrm{C} ;{ }^{1} \mathrm{H}$ NMR $(500 \mathrm{~Hz}$, $\left.\mathrm{CDCl}_{3}\right) \delta: 8.30(\mathrm{~s}, 1 \mathrm{H}, \mathrm{CH}=\mathrm{N}), 7.81 \sim 7.77(\mathrm{~m}, 2 \mathrm{H}, \mathrm{PhH})$, $7.45 \sim 7.42(\mathrm{~m}, 2 \mathrm{H}, \mathrm{PhH}), 3.73(\mathrm{t}, J=5.00 \mathrm{~Hz}, 2 \mathrm{H}$, $\mathrm{CH}_{2} \mathrm{OCH}_{2}$ ), 3.54 (t, J=4.75 Hz, 2H, $\left.\mathrm{CH}_{2} \mathrm{NCH}_{2}\right), 2.85$ (t, $\left.J=7.20 \mathrm{~Hz}, 2 \mathrm{H}, \mathrm{CH}_{2}\right), 1.81\left(\mathrm{t}, J=7.20 \mathrm{~Hz}, 2 \mathrm{H}, \mathrm{CH}_{2}\right), 1.36$ (t, $J=7.00 \mathrm{~Hz}, 4 \mathrm{H}, \mathrm{CH}_{2}$ ), 0.90 (t, $J=7.00 \mathrm{~Hz}, 3 \mathrm{H}, \mathrm{CH}_{3}$ ); ${ }^{13} \mathrm{C}$ NMR (125 MHz, $\mathrm{CDCl}_{3}$ ) $\delta: 176.64$ (triazine), 163.06 (triazole), 162.60 (triazole), $161.40(\mathrm{Ph}), 160.78$ (triazine), 150.82(N=CH), $130.74(\mathrm{Ph}), 129.04(\mathrm{Ph}), 116.28(\mathrm{Ph})$, 65.10 (morpholine), 43.20 (morpholine), 31.24 (alkyl), 25.84 (alkyl), 25.00 (alkyl), 22.14 (alkyl), 13.84 (alkyl); IR (KBr) v: 3030, 2925, 2850, 1686, 1600, 1500, 1450, 1380, 1290, 1250, $750 \mathrm{~cm}^{-1}$; HRMS (positive-ESIMS) calcd for $\mathrm{C}_{35} \mathrm{H}_{41} \mathrm{Cl}_{2} \mathrm{~N}_{12} \mathrm{OS}_{2}(\mathrm{M}+1)^{+}$779.2259, found 779.2267. 
二- $N$-(苯亚基)-3-((4-((4-((苯亚基)氨基-5-苯基4H-1,2,4-三唑-3-基)硫醚)-6-吡咯烷-1,3,5-三嗪-2-基)硫 醚)-5-苯基-4H-1,2,4-三唑-4-胺(16): 浅黄色固体, 收率 68.8\%. m.p. $178.4 \sim 179.6{ }^{\circ} \mathrm{C} ;{ }^{1} \mathrm{H} \mathrm{NMR}\left(500 \mathrm{~Hz}, \mathrm{CDCl}_{3}\right)$ $\delta: 8.24(\mathrm{~s}, 1 \mathrm{H}, \mathrm{CH}=\mathrm{N}), 7.96(\mathrm{~d}, J=8.75 \mathrm{~Hz}, 2 \mathrm{H}, \mathrm{PhH})$, $7.11(\mathrm{~d}, J=8.75 \mathrm{~Hz}, 3 \mathrm{H}, \mathrm{PhH}), 3.73$ (t, $J=4.95 \mathrm{~Hz}, 2 \mathrm{H}$, $\mathrm{CH}_{2} \mathrm{~N}$ ), 3.55 (t, $\left.J=5.90 \mathrm{~Hz}, 2 \mathrm{H}, \mathrm{CH}_{2}\right), 2.85$ (t, $J=7.50 \mathrm{~Hz}$, $\left.2 \mathrm{H}, \mathrm{CH}_{2}\right), 1.81\left(\mathrm{t}, J=7.45 \mathrm{~Hz}, 2 \mathrm{H}, \mathrm{CH}_{2}\right), 1.38 \sim 1.32(\mathrm{~m}$, $\left.4 \mathrm{H}, \mathrm{CH}_{2}\right), 0.90\left(\mathrm{q}, J=7.50 \mathrm{~Hz}, 3 \mathrm{H}, \mathrm{CH}_{3}\right) ;{ }^{13} \mathrm{C}$ NMR $(125$ $\mathrm{MHz}, \mathrm{CDCl}_{3}$ ) $\delta$ : 176.74 (triazine), 163.52 (triazole), 162.50 (triazole), 160.70 (triazine), $150.84(\mathrm{~N}=\mathrm{CH})$, $130.94(\mathrm{Ph}), 129.92(\mathrm{Ph}), 128.38(\mathrm{Ph}), 128.00(\mathrm{Ph}), 54.16$ (pyrrolidine), 31.20 (alkyl), 25.80 (alkyl), 25.24 (pyrrolidine), 25.01 (alkyl), 22.14 (alkyl), 13.85 (alkyl); IR (KBr) $v: 3030,2940,2850,1690,1600,1510,1450,1375,1270$, $876,745 \mathrm{~cm}^{-1}$; HRMS (positive-ESIMS) calcd for $\mathrm{C}_{35} \mathrm{H}_{43}$ $\mathrm{N}_{12} \mathrm{~S}_{2}(\mathrm{M}+1)^{+}$695.3088, found 695.3097.

二- $N$-(4-氯苯亚基)-3-((4-((4-((4-氯苯亚基)氨基-5苯基-4H-1,2,4-三唑-3-基)硫醚)-6-吡咯烷-1,3,5-三嗪-2基)硫醚)-5-苯基-4H-1,2,4-三唑-4-胺(17)：淡黄色固体, 收率 77.8\%. m.p. 205.7 206.6 ${ }^{\circ} \mathrm{C} ;{ }^{1} \mathrm{H}$ NMR $(500 \mathrm{~Hz}$, $\left.\mathrm{CDCl}_{3}\right) \delta: 8.24(\mathrm{~s}, 1 \mathrm{H}, \mathrm{CH}=\mathrm{N}), 7.88 \sim 7.85(\mathrm{~m}, 2 \mathrm{H}, \mathrm{PhH})$, $7.26 \sim 7.15(\mathrm{~m}, 2 \mathrm{H}, \mathrm{PhH}), 3.73\left(\mathrm{t}, J=4.95 \mathrm{~Hz}, 2 \mathrm{H}, \mathrm{CH}_{2} \mathrm{~N}\right)$, $3.55\left(\mathrm{t}, J=4.95 \mathrm{~Hz}, 2 \mathrm{H}, \mathrm{CH}_{2}\right), 2.85(\mathrm{t}, J=7.50 \mathrm{~Hz}, 2 \mathrm{H}$, $\mathrm{CH}_{2}$ ), $1.81\left(\mathrm{t}, J=7.45 \mathrm{~Hz}, 2 \mathrm{H}, \mathrm{CH}_{2}\right), 1.38 \sim 1.32(\mathrm{~m}, 4 \mathrm{H}$, $\left.\mathrm{CH}_{2}\right), 0.89$ (q, $\left.J=7.50 \mathrm{~Hz}, 3 \mathrm{H}, \mathrm{CH}_{3}\right) ;{ }^{13} \mathrm{C}$ NMR $(125 \mathrm{MHz}$, $\left.\mathrm{CDCl}_{3}\right) \delta: 176.64$ (triazine), 163.06 (triazole), 162.60 (triazole), $161.40(\mathrm{Ph}), 160.78($ triazine), $150.82(\mathrm{~N}=\mathrm{CH})$, $130.74(\mathrm{Ph}), 129.04(\mathrm{Ph}), 116.28(\mathrm{Ph}), 54.16$ (pyrrolidine), 31.24 (alkyl), 25.84 (alkyl), 25.24 (pyrrolidine), 25.00 (alkyl), 22.14 (alkyl), 13.84 (alkyl); IR (KBr) v: 3033, 2950, 2850, 1690, 1610, 1520, 1450, 1384, 1295, 830, $740 \mathrm{~cm}^{-1}$; HRMS (positive-ESIMS) calcd for $\mathrm{C}_{35} \mathrm{H}_{41} \mathrm{Cl}_{2} \mathrm{~N}_{12} \mathrm{~S}_{2}$ (M+ 1) ${ }^{+} 763.2311$, found 763.2317 .

\subsection{4目标化合物 $18 \sim 21$ 合成通法}

在氩气氛围下, 将 $0.5 \mathrm{mmol}$ 化合物(B1 或 B2) 和 0.2 $\mathrm{mmol}$ 四丁基澳化铵加入到西朗克管中, 向其中加入 10 $\mathrm{mL} \mathrm{POCl}_{3}$, 升温至 $90{ }^{\circ} \mathrm{C}$, 分批加入 $0.6 \mathrm{mmol}(\mathrm{A10}$ 或 A11), 加毕升温至 $100{ }^{\circ} \mathrm{C}$, 反应 $6 \mathrm{~h}$. 冷却, 减压蒸出 $\mathrm{POCl}_{3}$, 黄色油状物加入到 $100 \mathrm{~mL}$ 冰水中, 剧烈搅拌, 用 $\mathrm{K}_{2} \mathrm{CO}_{3}$ 和 $\mathrm{KOH}$ 的 $25 \%$ 的混合溶液调 $\mathrm{pH}$ 至 $6 \sim 7$, 静 置过夜, 过滤出沉淀物, 冷水洗涤, 乙醇和 DMF 混合溶 液重结晶, 干燥得到黄色固体产物 $\mathbf{1 8} \sim 21$.

4,6-双吗啉-2-(3-苯基-(1,2,4-三唑并噻二唑)-1,3,5三嗪(18): 黄色固体, 收率 $73 \%$. m.p. $230.6 \sim 231.3{ }^{\circ} \mathrm{C}$;
${ }^{1} \mathrm{H}$ NMR $\left(500 \mathrm{MHz}\right.$, DMSO- $\left.d_{6}\right) \delta: 9.65(\mathrm{~s}, 1 \mathrm{H}, \mathrm{NH}), 8.34$ $(\mathrm{d}, J=7.30 \mathrm{~Hz}, 2 \mathrm{H}, \mathrm{Ph}), 7.85(\mathrm{~d}, J=8.95 \mathrm{~Hz}, 2 \mathrm{H}, \mathrm{Ph})$, $7.65 \sim 7.57(\mathrm{~m}, 3 \mathrm{H}, \mathrm{Ph}), 6.87$ (d, $J=8.45 \mathrm{~Hz}, 2 \mathrm{H}, \mathrm{Ph}), 3.73$ (t, $J=4.70 \mathrm{~Hz}, 8 \mathrm{H}$, morpholine), $3.65(\mathrm{t}, J=4.70 \mathrm{~Hz}, 8 \mathrm{H}$, morpholine); ${ }^{13} \mathrm{C}$ NMR (125 MHz, DMSO- $\left.d_{6}\right) \delta: 172.61$ (triazine), 165.28 (triazine), 162.72 (triazole), 151.12 (triazole), 143.88 (thiadiazole), $138.93(\mathrm{Ph}), 131.82(\mathrm{Ph})$, $130.91(\mathrm{Ph}), 129.43(\mathrm{Ph}), 128.95(\mathrm{Ph}), 127.31(\mathrm{Ph}), 123.55$ $(\mathrm{Ph}), 116.82(\mathrm{Ph}), 66.38$ (morpholine), 44.71 (morpholine); IR (KBr) v: 3422, 3049, 2915, 1613, 1581, 1523, 1381, 1292, $1149 \mathrm{~cm}^{-1}$; HRMS (positive-ESIMS) calcd for $\mathrm{C}_{26} \mathrm{H}_{27} \mathrm{~N}_{10} \mathrm{O}_{2} \mathrm{~S}(\mathrm{M}+1)^{+}$543.2034, found 543.2035.

4,6-双四氢吡咯-2-(3-苯基-(1,2,4-三唑并噻二 唑)-1,3,5-三嗪(19): 黄色固体, 收率 71\%. m.p. 235.0 $235.9{ }^{\circ} \mathrm{C} ;{ }^{1} \mathrm{H}$ NMR $\left(500 \mathrm{MHz}, \mathrm{DMSO}-d_{6}\right) \delta: 9.65$ (s, $1 \mathrm{H}$, $\mathrm{NH}), 8.35(\mathrm{~d}, J=7.30 \mathrm{~Hz}, 2 \mathrm{H}, \mathrm{Ph}), 7.84(\mathrm{~d}, J=8.90 \mathrm{~Hz}$, $2 \mathrm{H}, \mathrm{Ph}), 7.65 \sim 7.57$ (m, 3H, Ph), 6.87 (d, $J=8.85 \mathrm{~Hz}, 2 \mathrm{H}$, $\mathrm{Ph}$ ), 3.59 (t, $J=6.85 \mathrm{~Hz}, 8 \mathrm{H}$, pyrrolidine), $1.98 \sim 1.96$ (m, 8H, pyrrolidine); ${ }^{13} \mathrm{C}$ NMR (125 MHz,DMSO- $\left.d_{6}\right) \delta$ : 172.61 (triazine), 165.28 (triazine), 162.72 (triazole), 151.12 (triazole), 143.88 (thiadiazole), $138.93(\mathrm{Ph}), 131.82$ $(\mathrm{Ph}), 130.91(\mathrm{Ph}), 129.43(\mathrm{Ph}), 128.95(\mathrm{Ph}), 127.31(\mathrm{Ph})$, $123.55(\mathrm{Ph}), 116.82(\mathrm{Ph}), 55.47$ (pyrrolidine), 25.51 (pyrrolidine); IR (KBr) v: 3423, 3061, 2914, 1613, 1581, 1515, 1386, $1293 \mathrm{~cm}^{-1}$; HRMS (positive-ESIMS) calcd for $\mathrm{C}_{26} \mathrm{H}_{27} \mathrm{~N}_{10} \mathrm{~S}(\mathrm{M}+1)^{+}$511.2045, found 511.2052.

4,6-双吗啉-2-(3-正戍基-(1,2,4-三唑并噻二唑)1,3,5-三嗪(20): 黄色固体，收率 80\%. m.p. 188.9 $189.4{ }^{\circ} \mathrm{C} ;{ }^{1} \mathrm{H}$ NMR $\left(500 \mathrm{MHz}, \mathrm{DMSO}-d_{6}\right) \delta: 9.62(\mathrm{~s}, 1 \mathrm{H}$, $\mathrm{NH}), 7.93(\mathrm{~d}, J=7.10 \mathrm{~Hz}, 2 \mathrm{H}, \mathrm{Ph}), 6.86(\mathrm{~d}, J=7.20 \mathrm{~Hz}$, $2 \mathrm{H}, \mathrm{Ph}$ ), 3.76 (t, $J=4.70 \mathrm{~Hz}, 8 \mathrm{H}$, morpholine), 3.67 (t, $J=$ $4.50 \mathrm{~Hz}, 8 \mathrm{H}$, morpholine), 2.79 (t, $J=7.50 \mathrm{~Hz}, 2 \mathrm{H}, \mathrm{CH}_{2}$ ), $1.63 \sim 1.59\left(\mathrm{~m}, 2 \mathrm{H}, \mathrm{CH}_{2}\right), 1.39 \sim 1.33\left(\mathrm{~m}, 4 \mathrm{H}, \mathrm{CH}_{2}\right), 0.90$ (t, $\left.J=7.50 \mathrm{~Hz}, 3 \mathrm{H}, \mathrm{CH}_{3}\right) ;{ }^{13} \mathrm{C}$ NMR (125 MHz,DMSO- $\left.d_{6}\right)$ $\delta: 172.62$ (triazine), 165.27 (triazine), 162.73 (triazole), 160.52 (triazole), 143.86(thiadiazole), $138.91(\mathrm{Ph}), 129.44$ (Ph), $123.55(\mathrm{Ph}), 116.82(\mathrm{Ph}), 66.38$ (morpholine), 44.71 (morpholine), 31.48 (alkyl), 25.80 (alkyl), 24.99 (alkyl), 22.16 (alkyl), 14.17 (alkyl); IR (KBr) v: 3429, 3033, 2931, 1622,1586, 1513 1380, 1294, $1153 \mathrm{~cm}^{-1}$; HRMS (positive-ESIMS) calcd for $\mathrm{C}_{25} \mathrm{H}_{33} \mathrm{~N}_{10} \mathrm{~S}(\mathrm{M}+1)^{+}$537.2433, found 537.2430.

4,6-双四氢吡咯-2-(3-正戊基-(1,2,4-三唑并噻二 唑)-1,3,5-三嗪(21)：黄色固体，收率 81\%. m.p. 199.8 $200.2{ }^{\circ} \mathrm{C} ;{ }^{1} \mathrm{H}$ NMR $\left(500 \mathrm{MHz}, \mathrm{CDCl}_{3}\right) \delta: 9.90(\mathrm{~s}, 1 \mathrm{H}$, 
NH), 7.99 (d, $J=8.30 \mathrm{~Hz}, 2 \mathrm{H}, \mathrm{Ph}), 6.68(\mathrm{~d}, J=8.60 \mathrm{~Hz}$, 2H, Ph), 3.69 (t, $J=6.45 \mathrm{~Hz}, 8 \mathrm{H}$, pyrrolidine), 2.89 (t, $J=$ $\left.7.50 \mathrm{~Hz}, 2 \mathrm{H}, \mathrm{CH}_{2}\right), 1.95 \sim 1.91(\mathrm{~m}, 8 \mathrm{H}$, pyrrolidine), $1.63 \sim 1.59\left(\mathrm{~m}, 2 \mathrm{H}, \mathrm{CH}_{2}\right), 1.40 \sim 1.35\left(\mathrm{~m}, 4 \mathrm{H}, \mathrm{CH}_{2}\right), 0.90$ $\left(\mathrm{t}, J=7.50 \mathrm{~Hz}, 3 \mathrm{H}, \mathrm{CH}_{3}\right) ;{ }^{13} \mathrm{C} \mathrm{NMR}\left(125 \mathrm{MHz}, \mathrm{CDCl}_{3}\right) \delta$ : 172.36 (triazine), 165.09 (triazine), 162.48 (triazole), 159.84 (triazole), 143.01 (thiadiazole), $138.63(\mathrm{Ph}), 129.43$ $(\mathrm{Ph}), 123.25(\mathrm{Ph}), 116.53(\mathrm{Ph}), 54.17$ (pyrrolidine), 31.18 (alkyl), 25.56 (pyrrolidine), 22.85 (alkyl), 22.03 (alkyl), 21.25 (alkyl), 14.01 (alkyl); IR (KBr) v: 3404, 3193, 2961, 1618, 1580,1523, 1381, $1274 \mathrm{~cm}^{-1}$; HRMS (positive-ESIMS) calcd for $\mathrm{C}_{25} \mathrm{H}_{33} \mathrm{~N}_{10} \mathrm{~S}(\mathrm{M}+1)^{+}$505.2510, found 505.2518

致谢 活性篎选试验由国家新药篮选中心协助测定, 在 此对他们的帮助表示诚挚的谢意.

辅助材料(Supporting Information) 化合物的核磁共 振氢谱和核磁共振碳谱. 这些材料可以免费从本刊网站 (http://sioc-journal.cn/)上下载.

\section{Referenes}

[1] Sharma, N.; Mohanakrishnan, D.; Shard, A.; Sharma, A.; Saima, A. K.; Sinha, D. Sahal J. Med. Chem. 2012, 55, 297.

[2] Saczewski, F.; Bułakowska, A. Eur. J. Med. Chem. 2006, 41, 611.

[3] Zheng, M.; Xu, C.; Ma, J.; Sun, Y.; Du, F.; Liu, H.; Lin, L.; Li, C.; Ding, J.; Chen, K.; Jiang, H. Bioorg. Med. Chem. 2007, 15, 1815.

[4] Liu, K.; Xia, B.; Ma, W.; Zheng, B.; Zhang, X.; Fan, B. QSAR Comb. Sci. 2008, 27, 425.

[5] El-Faham, A.; Soliman, S. M.; Ghabbour, H. A.; Elnakady, Y. A.; Mohaya, T. A.; Siddiqui, M. R. H. J. Mol. Struct. 2016, 1125, 121.

[6] Bennett, G. B.; Mason, R. B.; Alden, L. J.; RoachJr, J. B. J. Med. Chem. 1978, 21, 623.

[7] Ojha, H.; Gahlot, P.; Tiwari, A. K.; Pathak, M.; Kakkar, R. Chem. Biol. Drug Des. 2011, 77, 57.

[8] Agarwal, A.; Srivastava, K.; Puri, S. K.; Chauhan, P. M. S. Bioorg Med. Chem. Lett. 2005, 15(3), 531.

[9] Kapil, A.; Anshu, D. Bioorg. Med. Chem. 2007, 17, 3298.

[10] Mallon, R.; Feldberg, L. R.; Lucas, J.; Chaudhary, I.; Dehnhardt, C.; Santos, E. D.; Chen, Z.; Dos Santos, O.; Ayral-Kaloustian, S.; Venkatesan, A.; Hollander, I. Clin. Cancer Res. 2011, 17, 3193.

[11] Burger, M. T.; Pecchi, S.; Wagman, A.; Ni, Z. J.; Knapp, M.; Hendrickson, T.; Atallah, G.; Pfister, K.; Zhang, Y.; Bartulis, S.; Frazier, K.; Ng, S.; Smith, A.; Verhagen, J.; Haznedar, J.; Huh, K.; Iwanowicz, E.; Xin, X.; Menezes, D.; Merritt, H.; Lee, I.; Wiesmann, M.; Kaufman, S.; Crawford, K.; Chin, M.; Bussiere, D.; Shoemaker, K.; Zaror, I.; Maira, S. M.; Voliva, C. F. ACS. Med. Chem. Lett. 2011, 2, 774.

[12] Maira, S. M.; Pecchi, S.; Huang, A.; Burger, M.; Knapp, M.; Sterker, D.; Schnell, C.; Guthy, D.; Nagel, T.; Wiesmann, M.; Brachmann, S.; Fritsch, C.; Dorsch, M.; Chene, P.; Shoemaker, K.; Pover, A. D.; Menezes, D.; Martiny-Baron, G.; Fabbro, D.; Wilson, C. J.; Schlegel, R.; Hofmann, F.; Garcia- Echeverria, C.; Sellers, W. R.; Voliva, C. F. Mol. Cancer. Ther. 2012, 11, 317

[13] Sarıpınar, E.; Gecen, N.; Sahin, K.; Yaamaz, E. Eur. J. Med. Chem. 2010, 45(9), 4157

[14] Sunduru, N.; Gupta, L.; Chaturvedi, V.; Dwivedi, R.; Sinh, S.;
Chauhan, P. M. S. Eur. J. Med. Chem. 2010, 45(8), 3335.

[15] Kong, D. X.; Yamori, I. Acta Pharmacol. Sin. 2010, 31, 1189

[16] Badr, S. M. I.; Barwa, R. M. Bioorg. Med. Chem. 2011, 19, 4506.

[17] Goswami, B. N.; Kattaky, J. C. S.; Baruah, J. N. J. Heterocycl. Chem. 1984, 21, 1225.

[18] Seelam, N.; Shrivastava, S. P.; Prasanthi, S.; Gupta, S. J. Saudi Chem. Soc. 2016, 20, 411.

[19] Papakonstantinou-Garoufalias, S. S.; Tani, E.; Todoulou, O.; Papadaki-Valiraki, A.; Filippatos, E.; De Clercq, E.; Kourounakis, P. N. J. Pharm. Pharmacol. 1998, 50, 117.

[20] Turan-Zitouni, G.; Kaplancikli, Z. A.; Erol, K.; Kilic, F. S. Farmaco $1999,54,218$.

[21] Zhang, Z. Y.; Sun, X. W.; Chu, C. H.; Zhao, L. J. Chin. Chem. Soc. $1997,44,535$.

[22] Amir, M.; Harish, K.; Javed, S. A. Eur. J. Med. Chem. 2008, 43, 2056.

[23] Rzeski, W.; Matysiak, J.; Kandefer-Szerszen, M. Bioorg. Med. Chem. 2007, 15, 3201 .

[24] Karabasanagouda, T.; Adhikari, A. V.; Shettey, N. S. Eur. J. Med. Chem. 2007, 42, 521

[25] Padmavathi, V.; Reddy, G. S.; Padmaja, A.; Kondaiah, P.; Shazia, A. Eur. J. Med. Chem. 2009, 44, 2106.

[26] Shiradkar, M. R.; Padhalingappa, M. B.; Bhetalabhotala, S.; Akula, K. C.; Tupe, D. A.; Pinninti, R. R.; Thummanagoti, S. Bioorg. Med. Chem. 2007, 15, 6397.

[27] Webster, K. R.; Kimball, S. D.; Misra, R. N.; Xiao, H. Y.; Kim, K. S.; Lu, S. F.; Han, W. C.; Barbosa, S. A.; Hunt, J. T.; Rawlins, D. B.; Shan, W. F.; Ahmed, S. Z.; Qian, L. G.; Chen, B. C.; Zhao, R. L.; Bednarz, M. S.; Kellar, K. A.; Mulheron, J. G.; Batorsky, R.; Roongta, U.; Kamath, A.; Marathe, P.; Ranadive, S. A.; Sack, J. S.; Tokarski, J. S.; Pavletich, N. P.; Lee, F. Y. F. J. Med. Chem. 2004, 47, 1719.

[28] Misra, R. N.; Xiao, H.; Y.; Williams, D. K.; Kim, K. S.; Lu, S. F.; Keller, K. A.; Mulheron, J. G.; Batorsky, R.; Tokarski, J. S.; Sack, J. S.; Kimball, S. D.; Lee, F. Y.; Webster, K. R. Bioorg. Med. Chem. Lett. 2004, 14, 2973.

[29] Badr, S. M. I.; Barwa, R. M. Bioorg. Med. Chem., 2011, 19, 4506.

[30] Kumar, G. V. S.; Rajendraprasad, Y.; Mallikarjuna, B. P.; Chandrashekar, S. M.; Kistayya, C. Eur. J. Med. Chem. 2010, 45, 2063.

[31] Sui, Z. H.; Guan, J.; Hlasta, D. J.; Macielag, M. J.; Foleno, B. D.; Goldschmidt, R. M.; Loeloff, M. J.; Webb, G. C.; Barrett, J. F. Bioorg. Med. Chem. Lett. 1998, 8, 1929.

[32] Barton, B.; Gouwns, S.; Schaefer, M. C.; Zeelie, B. Org. Process Res. Dev. 2003, 7, 1071.

[33] Azenha, M. E. D.; Burrows, H. D.; Canle, M. L.; Coimbra, R.; Fernandez, M. I.; Garcia, M. V.; Rodrigues, A. E.; Santaballa, J. A.; Steenken, S.; Santaballa, J. A. Chem. Commun. 2003, 112.

[34] Zhang, C. L.; Guo, Y.; Sun, L. J.; Wu, Y. F.; Zhu, C. A.; Qu, R. F.; Wang, X.; Chai, J. H. Chin. J. Appl. Chem. 2014, 31, 1419 (in Chinese).

(张成路，国阳，孙立杰，武一菲，朱长安，曲瑞峰，王雪，柴金 华, 应用化学, 2014, 31, 1419.)

[35] Zhang, C. L.; Wang, X; Hu, X.; Sun, L. J.; Qu, R. F.; Guo, Y.; Cai, J. H.; Zhu, C. A. Chem. J. Chin. Univ. 2015, 36, 463 (in Chinese). (张成路, 王雪, 胡雪, 孙立杰，曲瑞峰，国阳，柴金华，朱长安， 高等学校化学学报, 2015, 36, 463.)

[36] Zhang, C. L.; Tang, J.; Yin, L. Y.; Xi, H.; Guo, Y.; Sun, L. J. Chin. J. Org. Chem. 2016, 36, 358 (in Chinese). (张成路，唐杰，殷立莹，袭焕，国阳，孙立杰，有机化学，2016, 36, 358.)

[37] Lavecchia, A.; Giovanni, C. D.; Pesapane, A.; Montuori, N.; Ragno, P.; Martucci, N. M.; Masullo, M.; Vendittis, E. D. J. Med. Chem. 2012, 55, 4142 .

[38] Zhu, C. A. M.S. Thesis, Liaoning Normal University, Dalian, 2015 (in Chinese). (朱长安, 硕士论文, 辽宁师范大学, 大连, 2015.)

[39] Chai, J. H. M.S. Thesis, Liaoning Normal University, Dalian, 2015 (in Chinese). 
(柴金华, 硕士论文, 辽宁师范大学, 大连, 2015.)

[40] Sun, L. J. M.S. Thesis, Liaoning Normal University, Dalian, 2016 (in Chinese).

(孙立杰, 硕士论文, 辽宁师范大学, 大连, 2016.)

[41] Zhang, R. B.; Lu, J. R.; Xin, C. W.; Liu, J. B.; Mu, J. B.; Yang, X. Y.; Wang, H. Y.; Wang, M. J.; Zhang, H. Chin. J. Org. Chem.
2015, 35, 858 (in Chinese)

(张瑞波，卢俊瑞，辛春伟，刘金彪，穆江蓓，杨旭云，王宏皿， 王美君，张贺，有机化学, 2015, 35, 858.)

[42] Wang, B. L.; Shi, Y. X.; Zhan, Y. Z.; Zhang, L. Y.; Zhang, Y.; Wang, L. Z.; Zhang, X.; Li, Y. H.; Li, Z. M.; Li, B. J. Chin. J. Chem. 2015 33,1124 .

(Li, L.; Lu, Z.) 